\title{
Semantic-Based Assembly Precision Optimization Strategy Considering Assembly Process Capacity
}

\author{
Xiaolin Shi ${ }^{1}\left(\right.$, Xitian Tian $^{1, *}$, Gangfeng Wang ${ }^{2}\left(\mathbb{D}\right.$ and Dongping Zhao ${ }^{3}$ \\ 1 Institute of Intelligent Manufacturing, School of Mechanical Engineering, \\ Northwestern Polytechnical University, Xi'an 710072, China; sx186@mail.nwpu.edu.cn \\ 2 Key Laboratory of Road Construction Technology and Equipment of MOE, School of Construction Machinery, \\ Chang'an University, Xi'an 710064, China; wanggf@chd.edu.cn \\ 3 School of Aircraft Engineering, Xi'an Aeronautical University, Xi'an 710077, China; 201612002@xaau.edu.cn \\ * Correspondence: tianxt@nwpu.edu.cn; Tel.: +86-29-8849-5445
}

check for

updates

Citation: Shi, X.; Tian, X.; Wang, G.; Zhao, D. Semantic-Based Assembly Precision Optimization Strategy Considering Assembly Process Capacity. Machines 2021, 9, 269. https://doi.org/10.3390/ machines 9110269

Received: 20 August 2021 Accepted: 1 November 2021 Published: 4 November 2021

Publisher's Note: MDPI stays neutral with regard to jurisdictional claims in published maps and institutional affiliations.

Copyright: (c) 2021 by the authors. Licensee MDPI, Basel, Switzerland. This article is an open access article distributed under the terms and conditions of the Creative Commons Attribution (CC BY) license (https:/ / creativecommons.org/licenses/by/ $4.0 /)$.

\begin{abstract}
Assembly precision optimization is an important means to ensure product accuracy, including two aspects: on the one hand, the relevant deviations of out-of-tolerance key characteristics are reduced to the design tolerance range; on the other hand, the deviation fluctuation range of key characteristics with a large process capability index $(\mathrm{Cp})$ can be extended to achieve the balance between accuracy, process capacity, and production cost. By virtue of the accumulated experience, a fast solution can be provided for the out-of-tolerance problem. Therefore, a semantic-based assembly precision optimization method considering process capacity is proposed in this paper. By constructing an ontology model between $\mathrm{Cp}$ and optimization strategy, a reasonable assembly precision optimization strategy can be pushed based on product accuracy analysis results. Firstly, an assembly precision optimization semantic model is established by association between analysis results, out-of-tolerance key characteristics, assembly process, and tolerance adjustment defined with Web Ontology Language (OWL) assertions. Furtherly, according to different Cp corresponding to different assembly success rates, Semantics Web Rule Language (SWRL) rules based on Cp are constructed to the push optimization strategy. Finally, the effectiveness of the model is illustrated by an aircraft inner flap.
\end{abstract}

Keywords: assembly precision optimization; process capability index; ontology; semantic; SWRL

\section{Introduction}

With the increasingly fierce competition in the global market environment, product quality has attracted more and more attention and become the core competitiveness of products, which is related to whether enterprises can win the competition and market share in the end [1,2]. The manufacturing process is an important factor affecting product quality, including the part manufacturing stage and product assembly stage [3]. The enterprise production mode has changed from a one-stop production mode in the past to the present mode, which gradually reduces part manufacturing proportion through outsourcing. The assembly stage in the whole enterprise manufacturing procedure accounts for a larger and larger proportion, which becomes an important part of the product life cycle and the main process of product function and performance realization [4-6]. With the change in production mode, competitive enterprises can obtain the same production resources so that the influence of part manufacturing quality on the final product quality is gradually weakened, and the influence of assembly quality is continuously enhanced [7]. According to statistics, the assembly procedure accounts for approximately 50 percent of the total production hours and more than 20 percent of the total product costs [2]. For complex products, such as the Boeing 747, made up of millions of parts, assembly is a much larger proportion of the production process. Therefore, to a certain extent, the product and market competitive advantages of an enterprise are closely related to the 
assembly stage [2,8]. Assembly precision directly affects product assembly quality, so it is very important to carry out assembly precision analysis and optimization [9-12].

High precision of products is one of the development trends in the machinery manufacturing industry, such as aircraft, automobiles, precision machining centers, and other products for high precision requirements [13,14]. Many factors affecting product assembly precision include not only part dimension and geometric tolerance change but also adjustment of the assembly process, etc. $[15,16]$. The final assembly accuracy of products is dependent on machining deviations of components and deviation accumulation in the assembly process, which are, respectively, determined by tolerance allocation in the design and adjustment processes in assembly [17,18]. Satisfying the increasingly higher accuracy requirement cannot only depend on tolerance specification. Measurements and adjustments are required for the assembly process to ensure the deviation accumulation is in a defined range. In each step of product assembly, deviations are accumulated due to incoming components with geometric deviations. Measurements are implemented to evaluate the deviations in key characteristics (KCs). Based on the measurement results, some critical characteristics should be adjusted to a relatively small value by scraping or remachining in order to obtain the target accuracy.

Due to the restriction of production cost in the actual design and production process, the tolerance range of parts cannot be reduced unlimitedly. In the process of product assembly, the assembly precision may not meet the design requirements due to the unreasonable assembly process design. Therefore, in the stage of assembly process design, assembly process design and assembly precision prediction and optimization are carried out alternately. Assembly process adjustment based on the prediction results of assembly accuracy is an effective method to optimize assembly accuracy, which can ensure the assembly accuracy of products to meet the design requirements in advance. It is more and more significant to consider the influence of part design tolerance and assembly process scheme for ensuring the balance between high precision and low cost by reducing tolerance requirements. According to the results of assembly precision analysis, design tolerance, assembly tolerance, and positioning tolerance, as well as assembly process parameters such as assembly sequence, positioning mode and positioning datum are optimized to achieve product assembly meeting precision requirements at a low manufacturing cost. The research status of assembly precision optimization technology is discussed from two aspects of assembly precision optimization model and optimization algorithm.

The existing assembly precision optimization models mainly take assembly cost, quality loss, and assembly precision as optimization objectives to realize the optimization of manufacturing tolerance and assembly tolerance. The assembly cost is inversely proportional to the part manufacturing tolerance and directly proportional to the manufacturing precision. Speckhart [19] first proposed the negative exponential model between assembly cost and part tolerance, which realized the optimization of dimensional tolerance under the constraint of assembly cost. Singh et al. [20] proposed a comprehensive method for simultaneously selecting design and manufacturing tolerances based on minimizing the total manufacturing cost. Wang et al. [21] proposed the aircraft assembly cost tolerance model, which takes into account the influence of assembly accuracy constraints, cost constraints, manufacturing accuracy constraints, and other constraints. Sanz-Lobera et al. [22] proposed the cost tolerance function of the manufacturing process. When the same part is selected in different manufacturing processes, the assembly cost will change. McKenna et al. [13] proposed a cost-dependent mutation propagation model for aircraft subcomponents over constraints. This model achieves a trade-off between manufacturing cost and the limits of achievable variation. Dong et al. [23] proposed an assembly precision optimization hybrid model by mixing the negative power precision optimization model and the exponential precision optimization model. The quality loss model is used to describe the influence of assembly precision on product quality and performance. Based on Taguchi's quality loss function, Jeang et al. [24] realized tolerance optimization design under the premise of minimum comprehensive cost. The above precision optimization model optimized part 
tolerance with assembly cost and quality as optimization objectives and improved product assembly precision to a certain extent. However, due to the limitation of actual manufacturing capacity, part tolerance optimization has some limitations. Taking assembly process parameters as optimization objects, assembly precision optimization can effectively ensure product assembly precision. Lu et al. [25] started from the influence of assembly sequence changes on positioning relationship of parts, determined assembly ability of parts, assembly precision, and accessibility of assembly function through assembly precision analysis, thus realizing assembly precision optimization. Martin et al. [26] focused on machine/process selection in sampling-based tolerance-cost optimization for dimensional tolerances considering the process capabilities and manufacturing distributions. Combining the assembly processing cost model with the accuracy, reliability, and tolerance principles, a tolerance optimization model of the static geometric accuracy was constructed [27].

With the in-depth study of intelligent algorithms, many researchers apply intelligent algorithms to tolerance allocation optimization design. Lee et al. [28] used a genetic algorithm (GA) and the process capability index to solve the robust objectives and probability constraints and to formulate a constrained optimization problem into an unconstrained one. Prabhaharan et al. [29] applied the continuous ant colony algorithm (CACO) as an optimization method to minimize the critical deviations and assign the optimal tolerance based on cost. Zhang et al. [30] proposed a linear programming method to optimize the tolerance distribution model. Wang et al. [31] proposed an improved heuristic search strategy to optimize the cost tolerance model. Kumar et al. [32] used the artificial bee colony algorithm and genetic algorithm to optimize the cost tolerance model. Kumar et al. [33] proposed a hybrid optimization algorithm combining tabu search and the metaheuristic algorithm. In addition, the optimization methods also include particle swarm optimization algorithm [34], simulated annealing algorithm [35], self-organizing migration algorithm [36], bat algorithm (BA) [37], game theory [38], etc. Balamurugan et al. [39] proposed that the differential evolution (DE) algorithm is superior to other optimization algorithms in the tolerance allocation model. Kumar et al. [40] used the genetic algorithm, nondominated sorting genetic algorithm (NSGA-II), and DE algorithm to optimize the cost tolerance model. Compared with the optimization result, the optimization effect of the DE algorithm is the best.

As mentioned above, some achievements have been made in the study of the assembly precision optimization model and the optimization algorithm, but these studies mainly take the tolerance as an optimization object, and the influence of assembly process parameters on assembly precision has not been paid enough attention. For complex products, there are many factors that affect the assembly precision, so it is difficult to establish an accurate precision optimization model in the assembly stage. In this case, it is of great significance to use the empirical knowledge/data corresponding to different precision analysis results to guide assembly precision optimization.

The assembly precision optimization process contains a wealth of expert experience and knowledge, and this expert experience and knowledge exist in the minds of technical personnel, which are the assembly production know-how and experience methods in the manufacturing field. Expert experience and knowledge can be used as important sources of assembly precision optimization knowledge after being explicit, because they are related to the long-term accumulated experience and knowledge of enterprises and the engineering experience suitable for the actual production situation of enterprises to seek a reasonable formal expression of optimization strategy, so that it can be recognized, stored, processed, and shared by computers. In the field of artificial intelligence, an ontology is defined as explicit formal specifications of shared conceptual models. "Shared" refers to the fact that ontology describes a commonly recognized concept in a domain and provides a common interpretation of the concept in the domain. "Conceptualization" refers to the abstraction of the objective world. "Explicit" means that concepts and their constraints are explicitly defined, and these definitions are not ambiguous. "Formalized" means that the representation of an ontology is both mathematically rigorous and computer 
readable and interpretable. The characteristics of an ontology can be used to realize semantic representation, intelligent reasoning, semantic exchange, and knowledge reuse in a real sense. Aiming at the problem of assembly precision optimization, the ontology theory and method are introduced into the research of assembly precision optimization. A semantic-based assembly precision optimization strategy considering the assembly process capacity approach is proposed in this paper. The semantic information of assembly precision optimization is represented by ontology, constraints and empirical knowledge are described by SWRL rules, and precision optimization is carried out on this basis.

On the basis of the above discussion, in order to improve the existing optimization models and methods in the field of assembly precision optimization, the proposed method has the following characteristics:

(1) By combining the empirical knowledge, the research on assembly precision optimization can quickly establish the assembly precision optimization strategy, which can be used to guide the assembly precision optimization.

(2) In the literature, the influence of multiple alternative assembly processes on precision optimization was rarely considered. The proposed method can be used to balance the cost and assembly accuracy of multiprocess complex assembly parts, and it is an effective precision optimization method.

(3) Manufacturing deviation in the process of parts machining is characterized by uncertainty and randomness. Tolerances of parts are gradually accumulated in mechanical assembly. The traditional analysis method did not consider the tolerance stack. The problem of tolerance stack is solved by the Monte Carlo simulation in the section of case study.

The rest of the paper is organized as follows. In Section 2, some basic concepts, principal, and procedures of our approach are provided. Section 3 establishes the assembly precision optimization semantic model and assembly precision optimization SWRL rules based on Cp. In Section 4, a case study is introduced to demonstrate the rationality of the proposed approach. Finally, we conclude the paper and present the future work in Section 5.

\section{Assembly Precision Optimization Based on Process Capability}

In the procedure of product design and process design, the precision of key characteristics is out of tolerance because of improper tolerance allocation or unreasonable formulation of the process scheme. Aiming at the problems of rework or rework that seriously affect the assembly efficiency and quality of products, the control method of product assembly precision is studied, and the assembly precision is analyzed, evaluated, and optimized in advance so as to ensure the assembly precision of products in advance in the assembly process preparation stage.

\subsection{Assembly Precision Optimization Principle}

Definition 1. Let $\Gamma\left(d_{\mathrm{i}}\right)$ be the assembly precision, where $d_{\mathrm{i}}(i=1,2, \cdots, n)$ are the $n$ kinds of deviations that constitute the deviation source. If any one of the deviations has a slight change, the ratio of assembly precision variation to deviation variation becomes the sensitivity, as shown in Equation (1).

$$
S_{i}=\frac{\Delta \Gamma}{\Delta d_{i}}(i=1,2, \cdots, n)
$$

where $S_{i}$ represents sensitivity, $\Gamma$ represents assembly precision associated with deviation source, $\Delta \Gamma$ represents slight variation in assembly precision, $d_{i}$ represents the $i$-th deviation affecting assembly precision, and $\Delta d_{i}$ represents slight variation in deviation source.

Definition 2. The actual assembly capacity of the assembly process under the control state in a certain period of time is the information source of the assembly precision optimization. Assembly process capacity is the ability of the assembly process to guarantee assembly quality, which is described by $C p$, as shown in Equation (2). 


$$
C p=\frac{\text { Design Requirements }}{\text { Assembly Capacity }}=\frac{U S L-L S L}{ \pm 3 \sigma}
$$

where $C p$ represents the process capability index, USL represents the upper limit of the given design tolerance, LSL represents the lower limit of the given design tolerance, and $\sigma$ represents the overall standard deviation of the design requirements reflecting the degree of assembly precision fluctuation.

In the procedure of predicting assembly precision, the deviation source and deviation accumulation collectively determine the product assembly precision, in which the design tolerance determines the magnitude of the deviation source, the deviation propagation path determines deviation accumulation, and the deviation propagation path is jointly determined by the process factors such as assembly sequence and positioning mode. Therefore, part design tolerance, assembly sequence, assembly tolerance, assembly process, tooling tolerance, tooling positioning method, and other information are taken as the input information of the assembly precision analysis. Based on the assembly constraint relationship, deviation propagation and accumulation are carried out to obtain the predictive result of the assembly precision of the key characteristics. According to the sensitivity $S_{i}$ of the assembly precision analysis result, the $\mathrm{Cp}$ corresponding to the out-of-tolerance key characteristics falls within the appropriate range by adjusting the input information of the assembly precision analysis so as to realize assembly precision optimization of the out-of-tolerance key characteristics. Assembly precision optimization is a complex problem that requires the product assembly to meet the design precision and performance requirements at the lowest possible manufacturing cost. Therefore, the assembly precision optimization problem takes the given performance requirements as the objective function and the assembly process capacity and tolerance domain specification as the constraint conditions, as shown in Equation (3).

$$
\left\{\begin{array}{l}
\Gamma \leq \Gamma_{A S M} \\
C p_{\min } \leq C p \leq C p_{\max } \\
L S L \leq T \leq U S L
\end{array}\right.
$$

where $\Gamma_{A S M}$ represents the given design precision requirements of the key characteristics, $C p_{\min }$ represents the lowest capability index of the existing assembly process, $C p_{\max }$ represents the highest capability index of the existing assembly process, and $T$ represents the deviation value of the deviation source.

According to the analysis of the assembly precision optimization principle, the assembly precision optimization process is a complex multiobjective solution process. In the process of assembly precision optimization, the efficiency of assembly precision optimization can be improved by effectively utilizing the accumulated empirical knowledge for a long time, and the targeted assembly precision optimization strategy can be provided for the relevant process designers. For enriching the semantics in assembly precision optimization and improving the reasoning capacity of the implicit knowledge underlying the existing accumulated data, an assembly precision optimization ontology modeling and reasoning framework based on $\mathrm{Cp}$ is proposed in this paper, taking advantage of the input information and the result of the assembly precision analysis. The framework is composed of two main parts:

(1) An assembly precision optimization semantic model depicting the terminology and data structure for assembly precision optimization.

(2) A reasoning mechanism based on $\mathrm{Cp}$ inferring the underlying relations among the existing data.

As illustrated in Figure 1, the information from the assembly precision analysis result is imported and stored as the ontology data in the proposed framework, which contains many implicit relations between the assembly precision analysis result and the assembly precision optimization strategy. On the strength of predefined rules constructed according to the assembly process knowledge, new relations and effective information will be inferred 
from the implicit relations to support efficient decision making for assembly precision optimization. These contents will be discussed in Section 3.

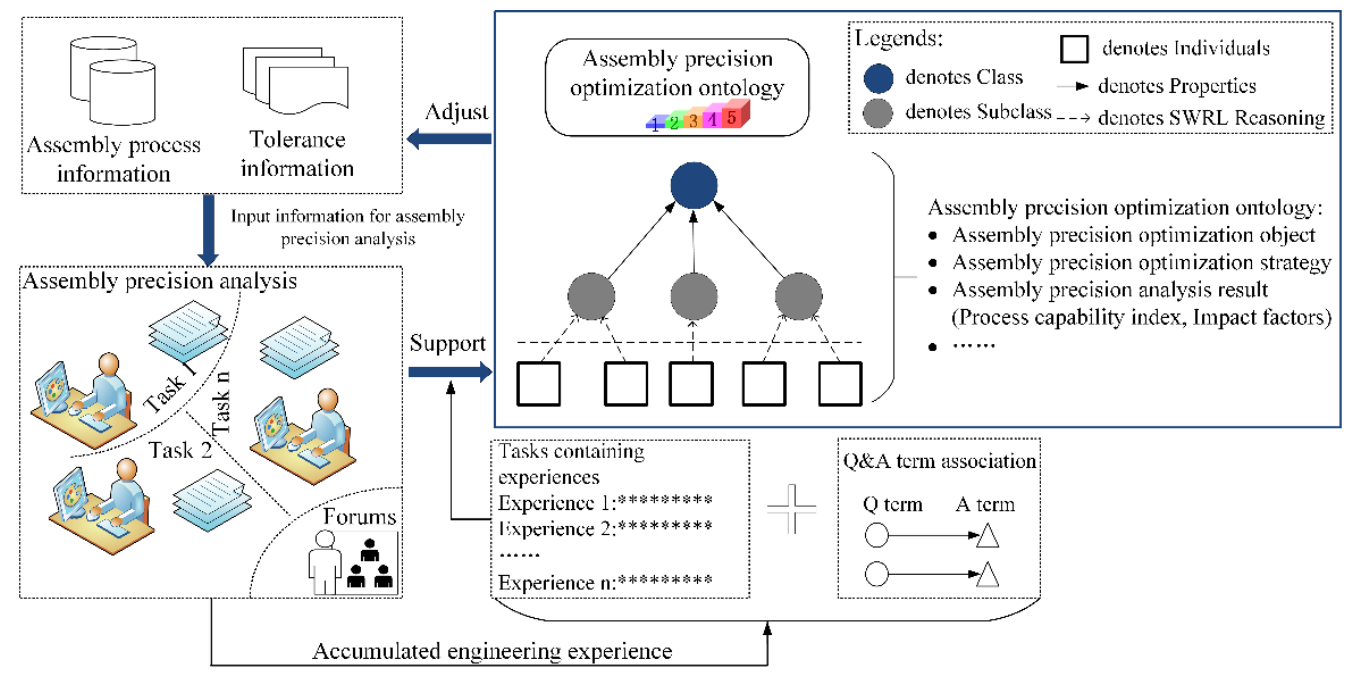

Figure 1. General framework of assembly precision optimization.

\subsection{Assembly Precision Optimization Procedure}

According to the above assembly precision optimization principle, assembly precision optimization objects mainly include the input information of the assembly precision analysis. The assembly process optimization object and tolerance information optimization object after precision optimization are used as input information to carry out assembly precision analysis verification until the precision of the key characteristics meets the design requirements. Assembly precision optimization can be implemented, as shown in Figure 2.

- Determination of key characteristics

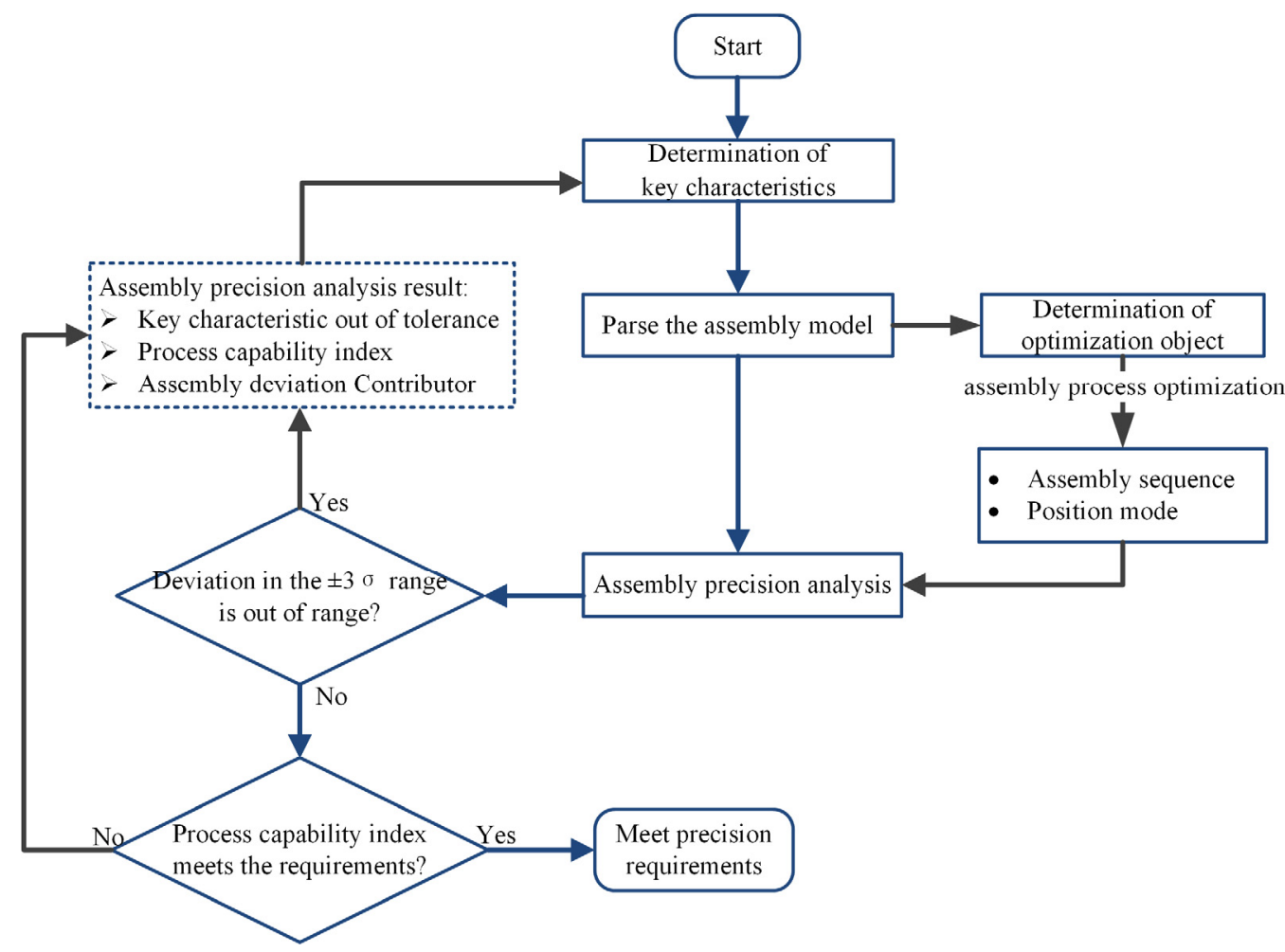

Figure 2. Assembly precision optimization procedure. 
The out-of-tolerance key characteristics that need to be optimized for assembly accuracy are determined based on the prediction results, functional requirements, structural characteristics, and assembly process scheme, which are used as the basis for parsing the associated assembly model. Out-of-tolerance key characteristics affect parameters related to assembly accuracies, such as distance accuracy, contact accuracy, mutual position accuracy, and mechanism motion accuracy.

- $\quad$ Parsing the assembly model

Through the prediction results, the out-of-tolerance key characteristics that need to be controlled are identified and the assembly model associated with key characteristics are further determined. The corresponding assembly model contains three-dimensional model information, assembly constraint information, assembly level information, assembly process information, design tolerance information, assembly tolerance information, etc. In order to reduce the calculation of assembly precision optimization, the assembly model is parsed. The assembly model information will be used as the input information of the assembly precision optimization. The assembly model, as the key link of the assembly precision optimization process, is used to quickly define the object range of assembly precision optimization.

- Assembly precision analysis

On the basis of the adjusted assembly process, the assembly precision analysis is carried out until the assembly accuracy meets the design requirements.

\section{An Ontology-Based Assembly Precision Optimization Model}

\subsection{Assembly Precision Optimization Semantic Model}

The semantic web and ontology theories are proposed to describe the semantic information and knowledge embedded in the real world. Ontology has sufficient ability to represent entities and their relations, as well as the knowledge structure of a particular domain. Many studies aim to apply semantic web and ontology theory to multiple fields such as knowledge sharing, semantic interoperability, and knowledge reuse [41-47]. Ontology modeling is the basis of constructing semantic applications. It is a unique advantage of the ontology model to use first-order predicate logic to carry out ontology reasoning and verification of concepts and their relations in the knowledge base based on ontology. With reference to the core product model $[48,49]$ and GD\&T specifications [50-53], the assembly precision optimization ontology is established in this section. It makes all the information semantically and formally in the decision system of assembly precision optimization.

Utilizing the Protégé ontology editor, the framework construction of a specific domain ontology can be decomposed into a series of definitions of classes, object properties, and data properties. Class is an abstract definition of a set of individuals in a certain domain: object properties express the binary relations among classes, and data properties express the unitary relations between classes and some specific data types, which provide varied descriptions to the classes. Figure 3 shows the construction procedure of an ontology aiming at assembly precision optimization for product precision analysis in the design stage.

- Step 1. Determining the domain and scope of the ontology application. In the first stage, some basic problems and concepts in the research field are determined, which help to understand the scope, definition domain, and comprehensiveness of ontology construction. Under the background of ensuring assembly precision in the product design stage, the established ontology application domain is to optimize assembly precision.

- Step 2. Considering the reusing of existing ontologies. Although ontology theory and technology have been applied in the design and manufacturing fields, such as assembly sequence planning, tolerance specification design, etc., it has not been applied in any assembly precision optimization-related domain. Therefore, no appropriate ontology can be reused.

- $\quad$ Step 3. Enumerating important terms in the ontology. In order to standardize this step, the main terms and their meanings are international standards and integrated 
scientific publications. Important terms include (1) terms related to assembly precision optimization, such as assembly precision optimization object, assembly precision optimization strategy, and (2) terms related to the assembly precision analysis result, such as assembly capacity level, assembly capacity level description, contributor, sensitivity, process capability index, and out-of-tolerance and within-design requirements. The listed terms above are used to semantically represent assembly precision analysis and optimization-related contents that constitute the application method of this study.

- Step 4. Definition of the classes and the class hierarchy. A composite development procedure is a combination of the top-down and bottom-up class construction approaches, the more salient concepts firstly being defined and then being generalized and specialized appropriately. A few top-level concepts such as assembly precision optimization and assembly precision analysis result are established and then related to a middle-level concept, such as assembly capacity level, assembly capacity level description, process capability index, assembly precision optimization object, and assembly precision optimization strategy. As shown in Figure 4, the hierarchy of the first- to fourth-level classes of assembly precision optimization ontology is presented.

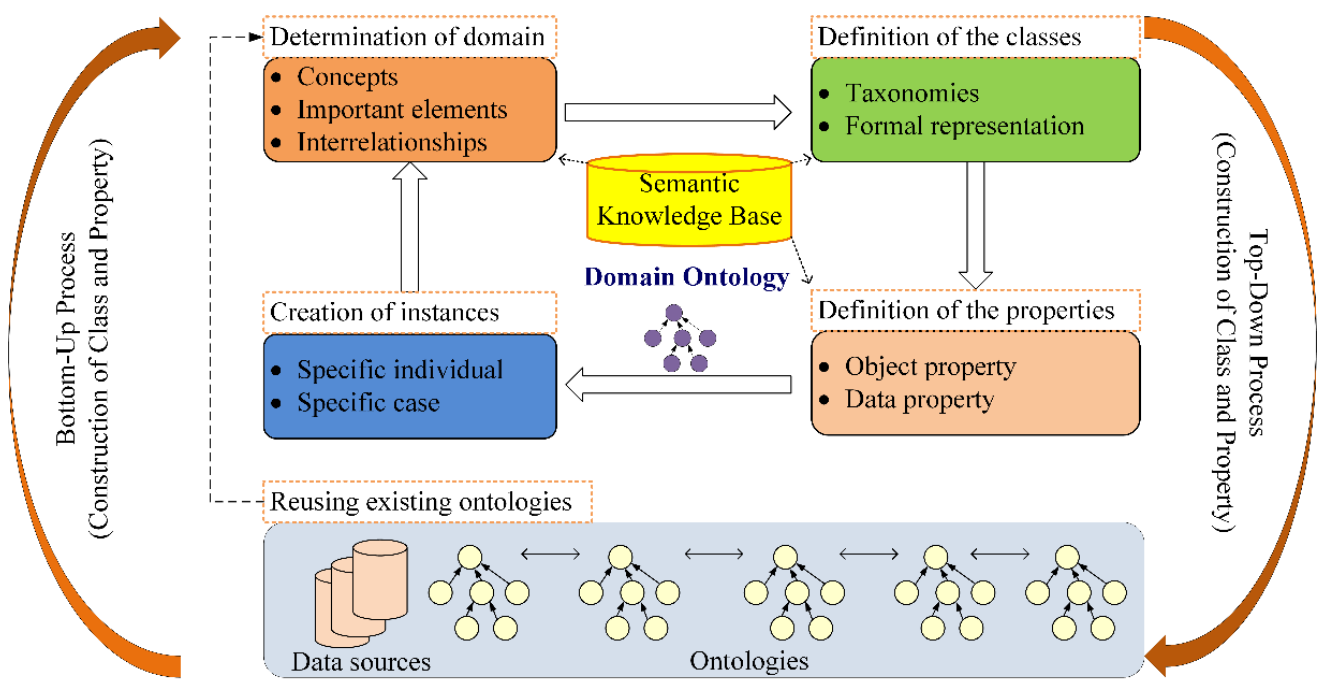

Figure 3. Basic modeling procedure for ontology establishment.

- Step 5. Definition of the properties of the classes. In the process of assembly precision optimization ontology construction, it is also necessary to describe the internal structure of concepts (classes) and their mutual relations for classes with defined top-level framework properties. As shown in Table 1, the object properties, their corresponding meanings, and their domain and range are listed. As the class definitions are refined and concretized, more property definitions (such as data property definitions) are added to the lower nonframework classes. The construction of classes and the definition of properties between classes are closely related, and the two processes occur interactively.

- $\quad$ Step 6. The last step of the assembly precision optimization ontology model is creating instances (also known as an object, value, or individual). The instances of classes are used to represent the specific problem of assembly precision optimization. The relationships between instances, classes, and properties are illustrated in Figure 5.

According to the assembly precision optimization principle, the information of assembly precision optimization can be organized as a semantic model, as shown in Figure 6. According to the results of assembly precision analysis, the process capability index, sensitivity, contributor, and other data are obtained to be used as an important basis for assembly precision optimization. The optimization range is determined according to the out-of-tolerance key characteristics. Different precision optimization strategies are adopted according to different ranges of process capability indexes. On the premise of satisfying the process 
capacity of the enterprise, the process optimization is given priority to achieve the balance between production cost and process capacity.

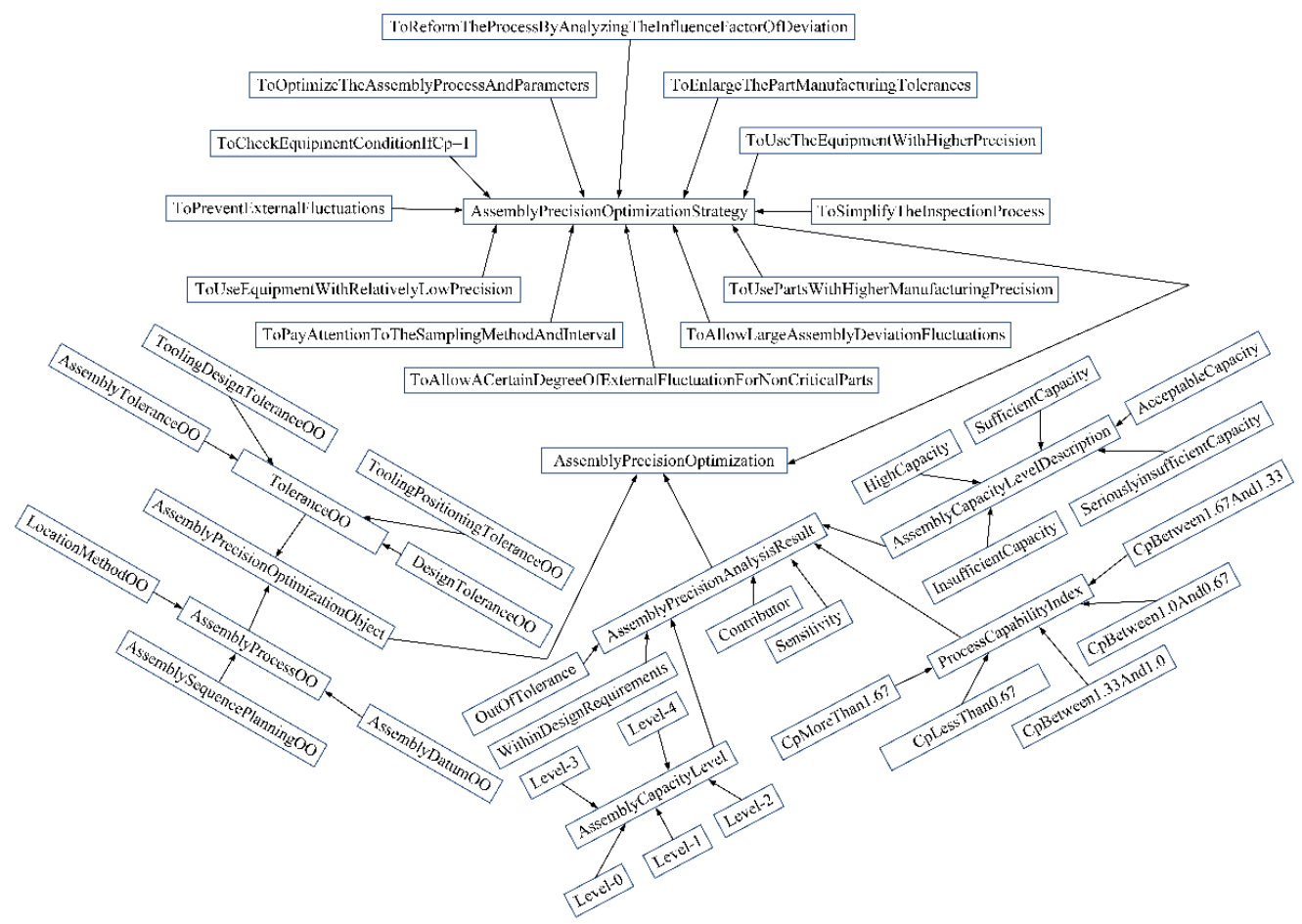

Figure 4. Hierarchy of classes in the ontology model.

Table 1. Object properties in the assembly precision optimization ontology.

\begin{tabular}{|c|c|c|c|c|}
\hline No. & Object Property & Meaning & Domains & Ranges \\
\hline 1 & $\begin{array}{c}\text { hasAssembly } \\
\text { PrecisionAnalysis }\end{array}$ & Assembly precision simulation is carried out & AssemblyModel & $\begin{array}{l}\text { AssemblyPrecision } \\
\text { AnalysisResult }\end{array}$ \\
\hline 2 & $\begin{array}{l}\text { hasProcess } \\
\text { CapabilityIndex }\end{array}$ & $\begin{array}{l}\text { Process capability index in the results of } \\
\text { assembly precision analysis }\end{array}$ & AssemblyModel & ProcessCapabilityIndex \\
\hline 3 & hasSensitivity & $\begin{array}{l}\text { The sensitivity of deviation source in the results } \\
\text { of assembly precision analysis }\end{array}$ & AssemblyModel & Sensitivity \\
\hline 4 & hasContributor & $\begin{array}{l}\text { The contributor of deviation source in the } \\
\text { results of assembly precision analysis }\end{array}$ & AssemblyModel & Contributor \\
\hline 5 & $\begin{array}{l}\text { hasKeyCharacteristics } \\
\text { OutOfTolerance }\end{array}$ & $\begin{array}{l}\text { Key characteristics out of tolerance in assembly } \\
\text { precision analysis }\end{array}$ & AssemblyModel & KeyCharacteristics \\
\hline 6 & $\begin{array}{l}\text { determineRangeOf } \\
\text { AssemblyPrecision } \\
\text { OptimizationObjects }\end{array}$ & $\begin{array}{l}\text { According to the results of assembly precision } \\
\text { analysis, the target range of assembly precision } \\
\text { optimization is determined }\end{array}$ & AssemblyModel & Subassembly \\
\hline 7 & $\begin{array}{l}\text { adjustAssembly } \\
\text { ProcessOutOf } \\
\text { Tolerance }\end{array}$ & $\begin{array}{l}\text { According to the determined optimization } \\
\text { object range, assembly process of the key } \\
\text { characteristics out of tolerance is adjusted }\end{array}$ & AssemblyModel & AssemblyProcess \\
\hline 8 & adjustDesign Tolerance & $\begin{array}{l}\text { According to the determined optimization } \\
\text { object range, design tolerance of the key } \\
\text { characteristics out of tolerance is adjusted }\end{array}$ & AssemblyModel & ToleranceInformation \\
\hline
\end{tabular}



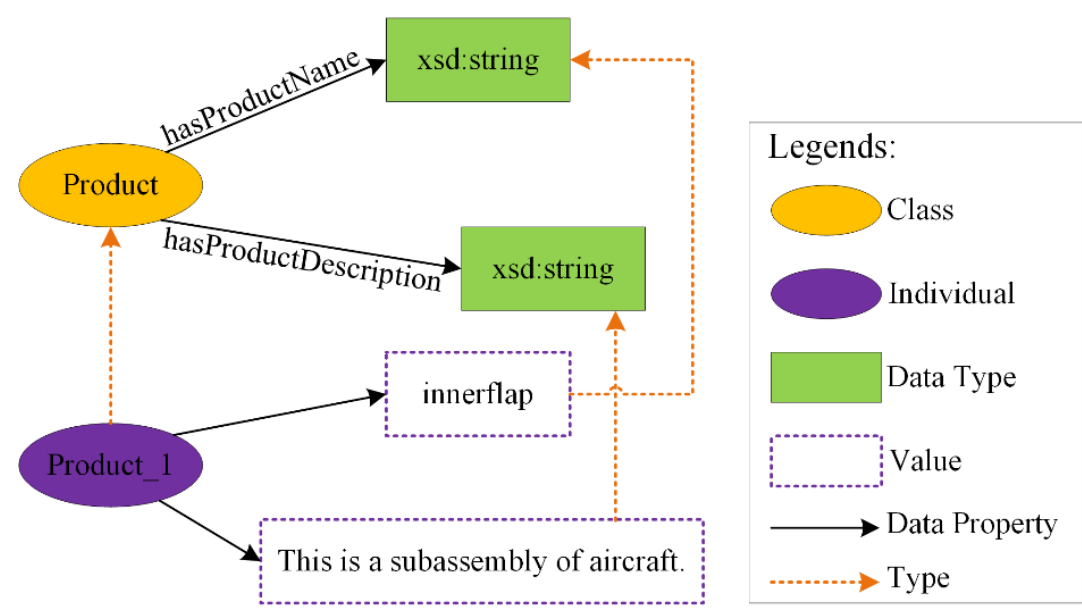

Figure 5. Relationships between instance, class, and property.

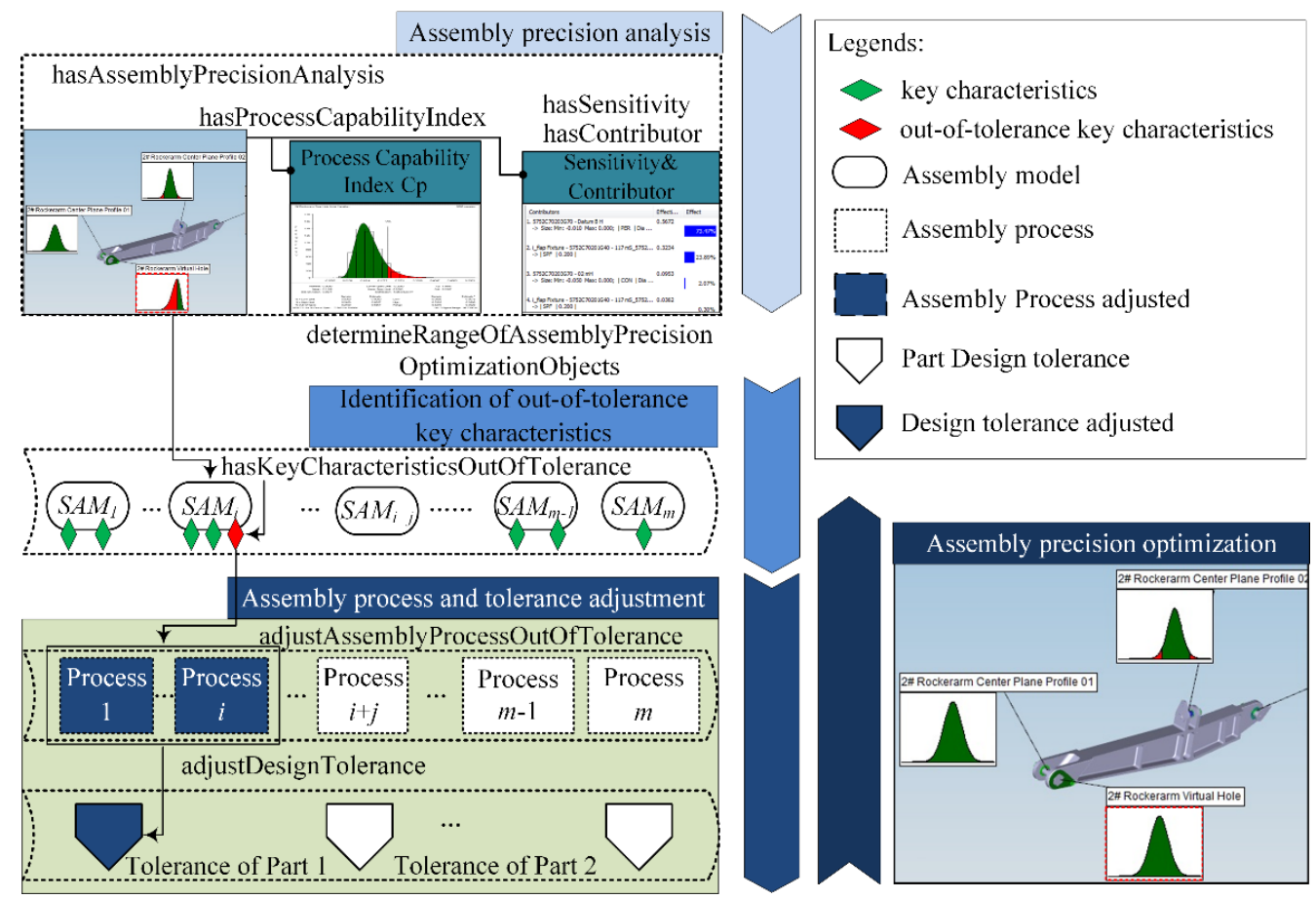

Figure 6. Assembly precision optimization semantic model.

\subsection{Assembly Precision Optimization SWRL Rules Based on Cp}

According to Definition 2 and Equation (2), Cp represents the relationship between the assembly process and design requirements indicating the ability of the assembly process to meet design requirements. According to the corresponding relationship between $C p$ and assembly success rate (Table 2), the assembly process capacity level is divided into five levels. Based on the analysis of process capacity at different levels and combined with long-term accumulated production experience, the following corresponding rules for fast determination of assembly precision optimization strategy are summarized, as illustrated in Table 3.

Table 2. Corresponding relationship between $\mathrm{Cp}$ and assembly success rate.

\begin{tabular}{cccccc}
\hline $\mathbf{C p}$ & 0.33 & 0.67 & 1.00 & 1.33 & 1.67 \\
\hline Assembly Success Rate & $68.27 \%$ & $95.45 \%$ & $99.73 \%$ & $99.993 \%$ & $99.999943 \%$ \\
\hline
\end{tabular}


Table 3. Assembly process capacity level division based on Cp.

\begin{tabular}{|c|c|c|c|c|}
\hline $\mathrm{Cp}$ & Level & Capacity & Distribution of the Sample & Relevant Methods \\
\hline$C p>1.67$ & Level 0 & High capacity & & $\begin{array}{l}\text { (1) Allow large deviation fluctuation } \\
\text { in assembly to improve efficiency; } \\
\text { (2) use less accurate equipment to } \\
\text { reduce manufacturing cost. }\end{array}$ \\
\hline $1.67 \geq \mathrm{Cp}>1.33$ & Level 1 & Sufficient capacity & & $\begin{array}{l}\text { (1) Magnify part manufacturing } \\
\text { tolerance; (2) allow some degree of } \\
\text { external fluctuation for noncritical } \\
\text { part; (3) simplify inspection process. }\end{array}$ \\
\hline $1.33 \geq \mathrm{Cp}>1.0$ & Level 2 & Acceptable capacity & & $\begin{array}{l}\text { (1) Protection against external } \\
\text { fluctuation; (2) pay attention to } \\
\text { sampling method and interval for } \\
\text { product sampling inspection; } \\
\text { (3) equipment condition should be } \\
\text { checked when } \mathrm{Cp}=1 \text {. }\end{array}$ \\
\hline $1.0 \geq \mathrm{Cp}>0.67$ & Level 3 & Insufficient capacity & & $\begin{array}{l}\text { (1) Optimize assembly process and } \\
\text { parameter; (2) use higher accurate } \\
\text { equipment to improve assembly } \\
\text { precision; (3) assembly with higher } \\
\text { manufacturing precision part. }\end{array}$ \\
\hline $0.67 \geq \mathrm{Cp}$ & Level 4 & $\begin{array}{l}\text { Seriously insufficient } \\
\text { capacity }\end{array}$ & & $\begin{array}{l}\text { (1) Analyze deviation contributor to } \\
\text { reform assembly process. }\end{array}$ \\
\hline
\end{tabular}

Rule 1: When the process capacity index is greater than 1.67 , the assembly capacity level is too high belonging to the super assembly capacity. In this case, the following two methods can be adopted: (1) allow large assembly deviation fluctuations; (2) use equipment with relatively low precision to reduce production cost and improve efficiency while ensuring the success rate of assembly.

Rule 2: When the process capacity index is within the range of 1.33 to 1.67 , the assembly capacity level is sufficient belonging to the first-level assembly capacity. In this case, the following three methods can be adopted: (1) enlarge the part manufacturing tolerances; (2) allow a certain degree of external fluctuation for noncritical parts; (3) simplify the inspection process to reduce production cost and improve efficiency while ensuring the success rate of assembly.

Rule 3: When the process capacity index is within the range of 1.0 to 1.33 , the assembly capacity level is acceptable belonging to the second-assembly capacity. In this case, the following three methods can be adopted: (1) prevent external fluctuations; (2) pay attention to the sampling method and interval during product sampling inspection; (3) check equipment condition if $\mathrm{Cp}=1$ to ensure the success rate of assembly.

Rule 4: When the process capacity index is within the range of 0.67 to 1.0 , the assembly capacity level is insufficient belonging to the third-level assembly capacity. In this case, the following three methods can be adopted: (1) optimize the assembly process and parameters; (2) use the equipment with higher precision; (3) use parts with higher manufacturing precision to ensure the success rate of assembly.

Rule 5: When the process capacity index is less than 0.67 , the assembly capacity is seriously insufficient belonging to the fourth level of assembly capacity. In this case, it is 
necessary to reform the process by analyzing the influence factor of deviation to ensure the success rate of assembly.

The SWRL rule language tightly integrating with OWL is used to express rule knowledge because the predicates of SWRL rules are consistent with classes and properties constructed by OWL. On account of the classes and properties that have been modeled in Protégé-OWL, mapping relations between assembly precision analysis results and assembly precision optimization strategies in Table 3 can be expressed in SWRL, as shown in Table 4.

Table 4. SWRL rules of characterization value determination.

\begin{tabular}{|c|c|}
\hline NO. & SWRL Rules \\
\hline $1-1$ & $\begin{array}{l}\text { hasAssemblyCapacity(?x,acl_Level-0), hasAssemblyCapacityDescription(?x,acld_HighCapacity), } \\
\text { hasProcessCapabilityIndex(?x,cp_CpMoreThan1.67) } \\
\text {->optimizeAssemblyPrecision(?x,apos_ToAllowLargeAssemblyDeviationFluctuations) }\end{array}$ \\
\hline $1-2$ & $\begin{array}{l}\text { hasAssemblyCapacity(?x,acl_Level-0), hasAssemblyCapacityDescription(?x,acld_HighCapacity), } \\
\text { hasProcessCapabilityIndex(?x,cp_CpMoreThan1.67) } \\
\text {->optimizeAssemblyPrecision(?x,apos_ToUseEquipmentWithRelativelyLowPrecision) }\end{array}$ \\
\hline $2-1$ & $\begin{array}{l}\text { hasAssemblyCapacity(?x,acl_Level-1), hasAssemblyCapacityDescription(?x,acld_SufficientCapacity), } \\
\text { hasProcessCapabilityIndex(?x,cp_CpBetween1.67And1.33) } \\
\text {->optimizeAssemblyPrecision(?x,apos_ToEnlargeThePartManufacturingTolerances) }\end{array}$ \\
\hline $2-2$ & $\begin{array}{l}\text { hasAssemblyCapacity(?x,acl_Level-1), hasAssemblyCapacityDescription(?x,acld_SufficientCapacity), } \\
\text { hasProcessCapabilityIndex(?x,cp_CpBetween1.67And1.33) } \\
\text {->optimizeAssemblyPrecision(?x,apos_ToAllowACertainDegreeOfExternalFluctuationForNonCriticalParts) }\end{array}$ \\
\hline $2-3$ & $\begin{array}{l}\text { hasAssemblyCapacity(?x,acl_Level-1), hasAssemblyCapacityDescription(?x,acld_SufficientCapacity), } \\
\text { hasProcessCapabilityIndex(?x,cp_CpBetween1.67And1.33) -> } \\
\text { optimizeAssemblyPrecision(?x,apos_ToSimplifyTheInspectionProcess) }\end{array}$ \\
\hline $3-1$ & $\begin{array}{l}\text { hasAssemblyCapacity(?x,acl_Level-2), hasAssemblyCapacityDescription(?x,acld_AcceptableCapacity), } \\
\text { hasProcessCapabilityIndex(?x,cp_CpBetween1.33And1.0) } \\
\text {->optimizeAssemblyPrecision(?x,apos_ToPreventExternalFluctuations) }\end{array}$ \\
\hline $3-2$ & $\begin{array}{l}\text { hasAssemblyCapacity(?x,acl_Level-2), hasAssemblyCapacityDescription(?x,acld_AcceptableCapacity), } \\
\text { hasProcessCapabilityIndex(?x,cp_CpBetween1.33And1.0) } \\
\text {->optimizeAssemblyPrecision(?x,apos_ToPayAttentionToTheSamplingMethodAndInterval) }\end{array}$ \\
\hline $3-3$ & $\begin{array}{l}\text { hasAssemblyCapacity(?x,acl_Level-2), hasAssemblyCapacityDescription(?x,acld_AcceptableCapacity), } \\
\text { hasProcessCapabilityIndex(?x,cp_CpBetween1.33And1.0) } \\
\text {->optimizeAssemblyPrecision(?x,apos_ToCheckEquipmentConditionIfCp =1) }\end{array}$ \\
\hline $4-1$ & $\begin{array}{l}\text { hasAssemblyCapacity(?x,acl_Level-3), hasAssemblyCapacityDescription(?x,acld_InsufficientCapacity), } \\
\text { hasProcessCapabilityIndex(?x,cp_CpBetween1.0And0.67) } \\
\text {->optimizeAssemblyPrecision(?x,apos_ToOptimizeTheAssemblyProcessAndParameters) }\end{array}$ \\
\hline $4-2$ & $\begin{array}{l}\text { hasAssemblyCapacity(?x,acl_Level-3), hasAssemblyCapacityDescription(?x,acld_InsufficientCapacity), } \\
\text { hasProcessCapabilityIndex(?x,cp_CpBetween1.0And0.67) } \\
\text {->optimizeAssemblyPrecision(?x,apos_ToUseTheEquipmentWithHigherPrecision) }\end{array}$ \\
\hline $4-3$ & $\begin{array}{l}\text { hasAssemblyCapacity(?x,acl_Level-3), hasAssemblyCapacityDescription(?x,acld_InsufficientCapacity), } \\
\text { hasProcessCapabilityIndex(?x,cp_CpBetween1.0And0.67) } \\
\text {->optimizeAssemblyPrecision(?x,apos_ToUsePartsWithHigherManufacturingPrecision) }\end{array}$ \\
\hline $5-1$ & $\begin{array}{l}\text { hasAssemblyCapacity(?x,acl_Level-4), hasAssemblyCapacityDescription(?x,acld_SeriouslyInsufficientCapacity), } \\
\text { hasProcessCapabilityIndex(?x,cp_CpLessThan0.67) } \\
\text {->optimizeAssemblyPrecision(?x,apos_ToReformTheProcessByAnalyzingTheInfluenceFactorOfDeviation) }\end{array}$ \\
\hline
\end{tabular}

\section{Case Study}

The effectiveness of the proposed approach is verified by an example of an aircraft inner flap. Because the inner flap is the key component of the aircraft, which plays a decisive role in the take-off and landing performance of the aircraft, the requirements for assembly precision are higher. The structure of the inner flap is shown in Figure 7, which is mainly composed of sliding rail, rocker arm, leading edge, middle segment, and trailing edge, including front and rear beams, torsion tube, slide rib, end rib, common rib, upper and lower wing surfaces, rocker arm joints, etc. When the inner flap rotates, the connecting rod provides power to drive the rocker arm, which drives the inner flap down through the front and rear joints. 


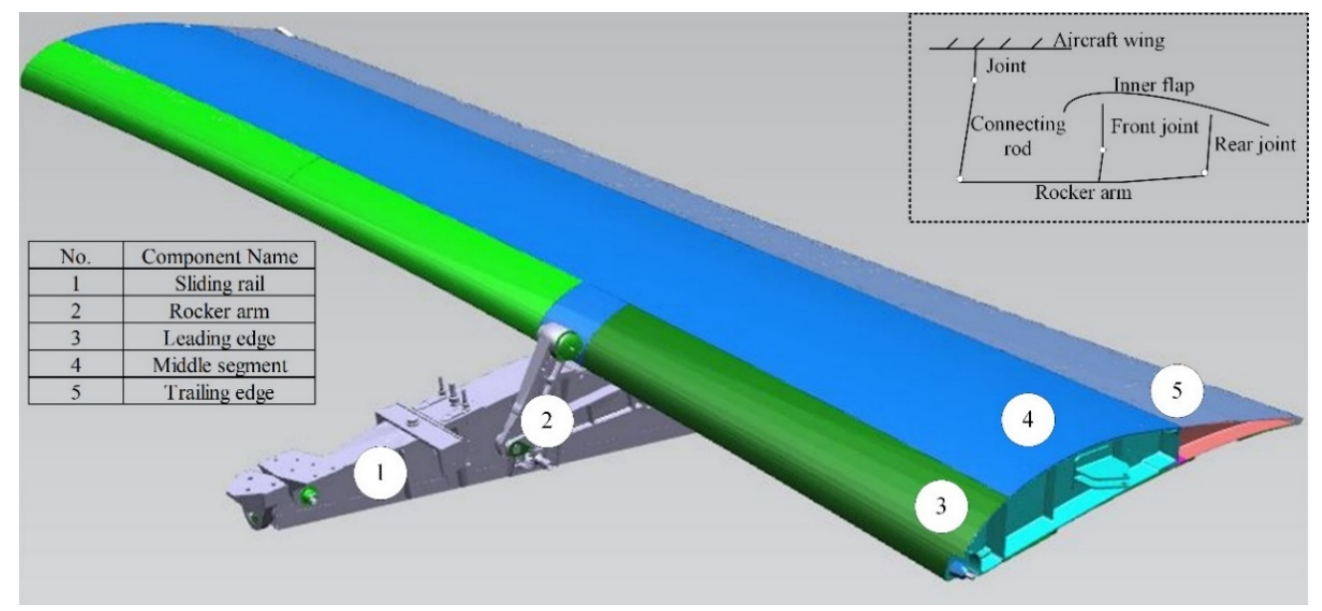

Figure 7. Inner flap geometric model.

- The Monte Carlo algorithm is used to calculate the cumulative results of assembly deviation. In order to make the statistical result closer to the true value, 5000 groups of random deviation values are taken for assembly precision analysis. The final assembly precision analysis result is shown in Figure 8. According to the result of the inner flap assembly precision analysis, the axis deviation of the rocker arm front intersection hole is out of tolerance, and the distribution of axis deviation is shown in Figure 8, in which the $\mathrm{Cp}$ of the key characteristic is 0.13 . According to Table 3 , the process capacity level of the key characteristic is seriously insufficient. The optimized assembly process information should be determined according to assembly deviation source impact factors so as to realize the assembly precision optimization of the front intersection hole axis deviation of the rocker arm. According to Figure 8, it can be seen that the main influencing factors of the front intersection hole axis deviation are: the floating of the rocker arm and front and rear joints caused by bolt assembly, deviation of the rocker arm rear joint assembling hole, deviation of the rocker arm rear joint assembly tooling, and deviation of the rocker arm assembly datum B.

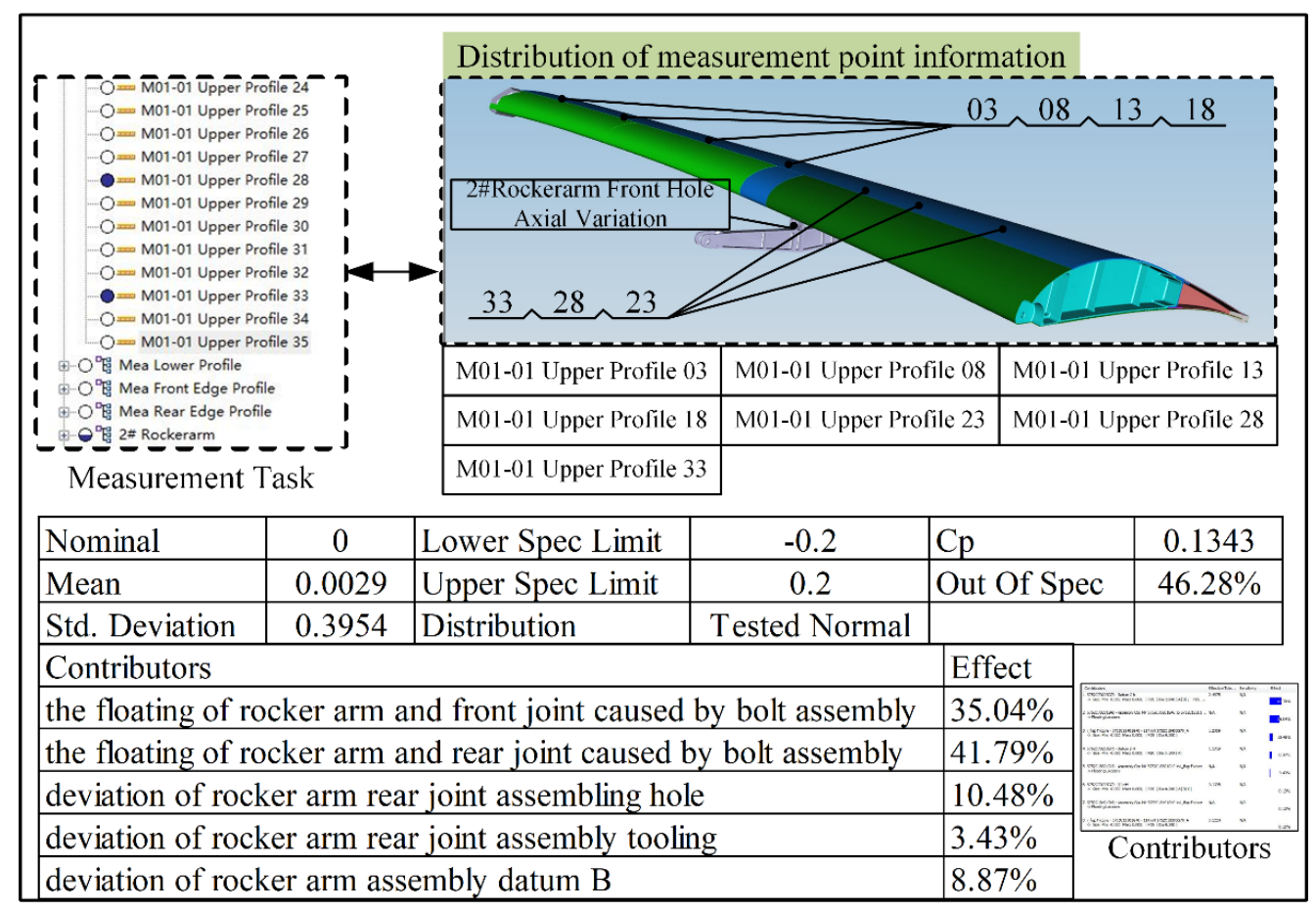

Figure 8. Assembly precision analysis result of inner flap. 
- According to the inference machine Hermit of Protege software, the results show that the assembly process needs to be reformed for achieving assembly precision optimization and meeting the requirements of assembly precision, as shown in Figure 9. The positioning mode of the front and rear joints and the deviation of the front and rear joints positioning tooling are the first targets to be optimized. The upper semantic framework of the proposed method is established through conceptualization. Next, concrete instances are built based on the defined concepts for semantic instance modeling of the analyzed products. The inference rules applicable to Protégé are established according to the SWRL rules mentioned in the third section. Finally, the inference result is obtained by running the inference machine, and the strategy for assembly precision optimization is obtained.

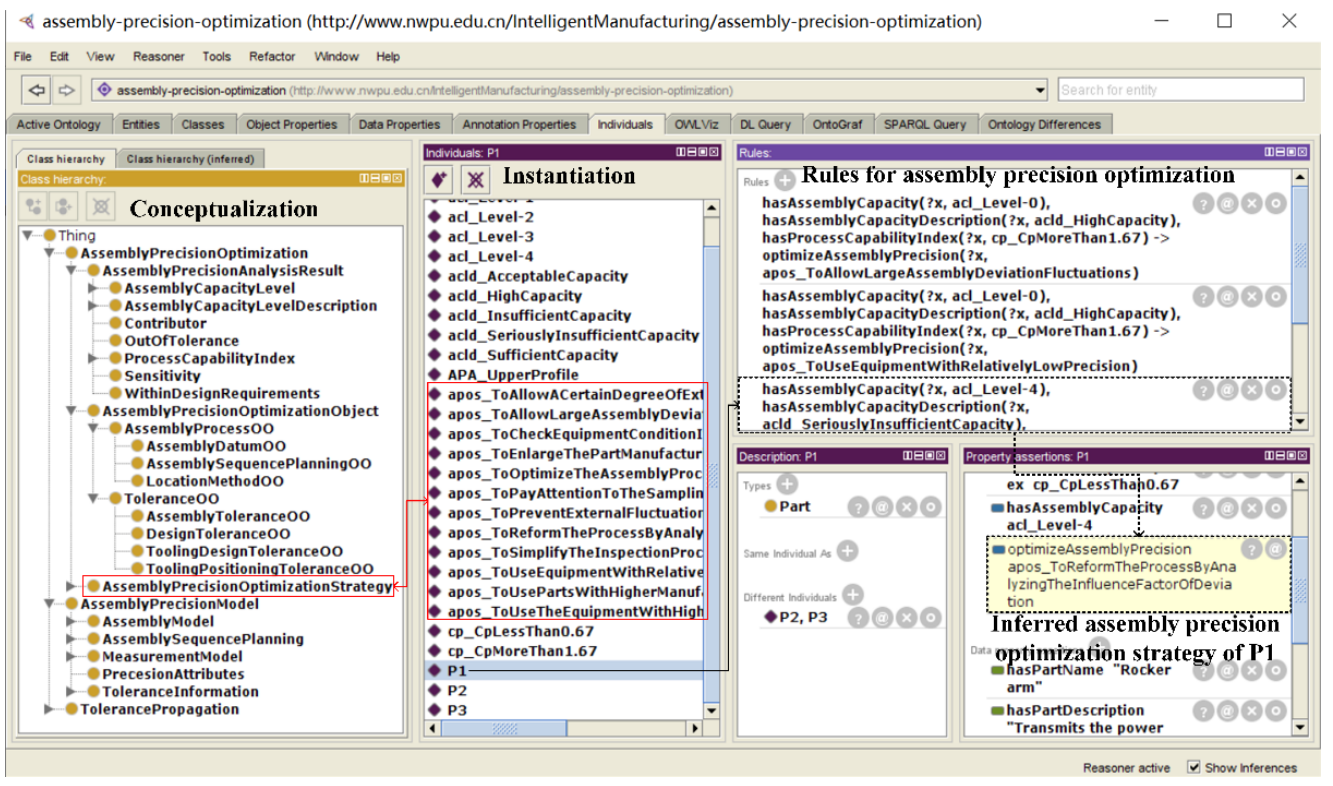

Figure 9. Instantiation of the pushed assembly precision optimization strategy.

- Before the optimization of the assembly process, the rocker arm and inner flap are assembled directly through the front and rear joints, which is conducive to the interchangeability of parts, as shown in Figure 10 (Scheme 1). In order to ensure assembly precision of the front intersection hole of the rocker arm, the front and rear joints of the rocker arm are assembled with tooling to directly locate the intersection hole of the parts and the surface where the intersection hole is located. The rocker arm positioning mode is changed to the front and rear intersection holes and rocker arm plane positioning, as shown in Figure 10 (Scheme 2). Through the optimization of the assembly process, the $\pm 3 \sigma$ value and $C p$ value are, respectively, 0.05 and 1.35 , which are within the target range of assembly process optimization, indicating that assembly process optimization has realized the precision optimization target of the front intersection hole axis deviation. A comparison between the original assembly process scheme (Scheme 1) and the assembly process optimization scheme (Scheme 2) is shown in Figure 10. 


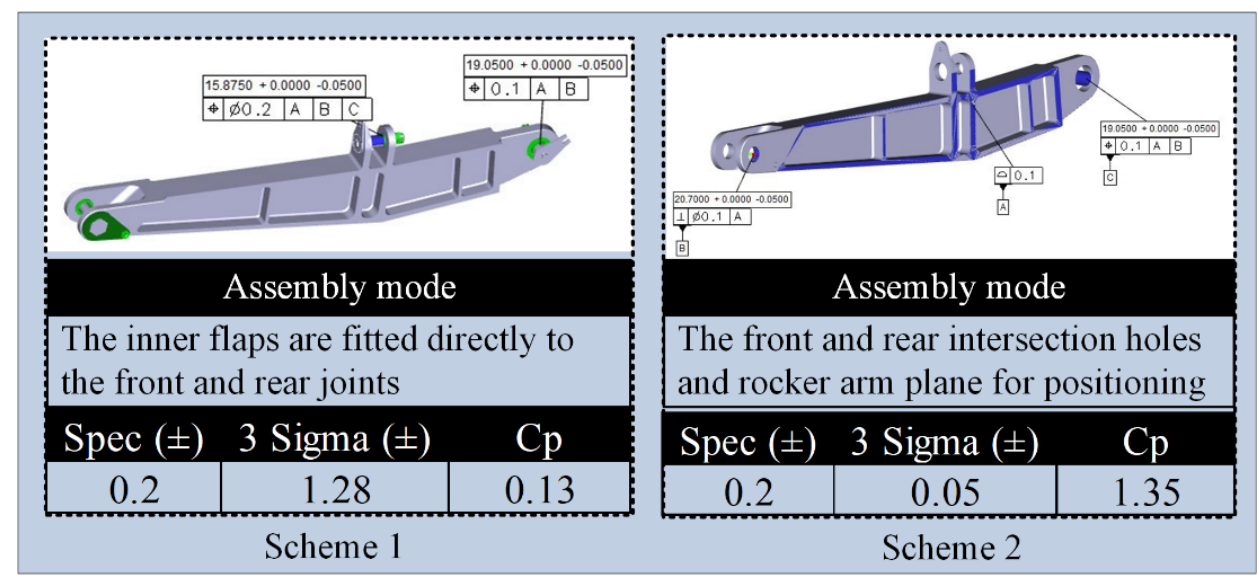

Figure 10. Schemes comparison.

\section{Conclusions}

In this paper, a structuralized modeling and reasoning framework based on ontology is presented for assembly precision optimization, which is used to build the semantic association model between engineering semantic knowledge and assembly precision optimization. Firstly, the assembly precision optimization semantic model was constructed based on multilevel descriptors, which were logically divided into the assembly precision analysis results, the out-of-tolerance key characteristics, and the assembly process and design tolerance adjustment. The results of the assembly precision analysis, the out-of-tolerance key characteristics, and the adjustment of the assembly process and design tolerance are related. Then, according to the accumulated effective engineering experience, the corresponding relationship between the assembly process capability index and the assembly process optimization is established. At the same time, the instantiation assembly model data are represented structurally based on ontology mapping. The main contributions of this research are as follows.

(1) Aiming at the problem that the product key characteristics are out of tolerance in the simulation results of assembly precision, the ontology theory and technology are introduced into the research of assembly precision optimization, and an assembly precision optimization technology based on process capability is proposed, which lays the foundation for subsequent computer-aided tolerance allocation and assembly process scheme optimization. The principle of assembly precision optimization based on process capability is analyzed, and the process of assembly precision optimization is explained. The corresponding relationship between process capability index and assembly precision optimization is summarized.

(2) The semantic model of assembly precision optimization, which has multilevel features of the assembly precision analysis results, out-of-tolerance key characteristics, and assembly process and design tolerance adjustment, is introduced as the bridge between product the CAD model and assembly precision guarantee. The class module and instance module of ontology are used to integrate the high-level semantic concepts of the assembly precision analysis system and the assembly precision analysis data of specific products into a model framework. The semantic model of assembly precision optimization is composed of several layers and elements. The semantic model of assembly precision optimization is extended according to different granularity, and it is used as the link of CAT and CAPP integration.

The potential research work is to furtherly explore the application of semantic methods in the field of assembly precision optimization. The assembly precision optimization semantic model can be expanded in consideration of more constraints and inference SWRL rules can be added to accommodate more complex products. Combined with the latest 
knowledge graph technology, it is expected to further improve the effectiveness of assembly precision optimization.

Author Contributions: Conceptualization, methodology, X.S. and X.T.; software, validation, X.S., G.W., and D.Z.; writing — original draft preparation, writing—review and editing, X.S. All authors have read and agreed to the published version of the manuscript.

Funding: This work was partially supported by the Natural Science Basic Research Project of Shaanxi Province, China (Grant Nos. 2019JM-073 and 2019JM-435) and the China Postdoctoral Science Foundation (Grant No. 2018M633439).

Institutional Review Board Statement: Not applicable.

Informed Consent Statement: Not applicable.

Data Availability Statement: Not applicable.

Conflicts of Interest: The authors declare no conflict of interest.

\section{References}

1. Demoly, F.; Yan, X.T.; Eynard, B.; Rivest, L.; Gomes, S. An assembly oriented design framework for product structure engineering and assembly sequence planning. Robot Comput. Integr. Manuf. 2011, 27, 33-46. [CrossRef]

2. Ou, L.M.; Xu, X. Relationship matrix based automatic assembly sequence generation from a CAD model. Comput. Aided Des. 2013, 45, 1053-1067. [CrossRef]

3. Masehian, E.; Ghandi, S. Assembly sequence and path planning for monotone and nonmonotone assemblies with rigid and flexible parts. Robot Comput. Integr. Manuf. 2021, 72, 102180. [CrossRef]

4. Li, R.K.; Hwang, C.L. A framework for automatic DFA system-development. Comput. Ind. Eng. 1992, 22, 403-413. [CrossRef]

5. Desai, A. Ease of product assembly through a time-based design methodology. Assem. Autom. 2019, 39, 881-903. [CrossRef]

6. Zhang, H.; Peng, Q.J.; Zhang, J.; Gu, P.H. Planning for automatic product assembly using reinforcement learning. Comput. Ind. 2021, 130, 103471. [CrossRef]

7. Ni, J.; Liu, R.; Sun, Y. Algorithm for Accumulating Part Mating Gaps to Evaluate Solid and Fluid Performances. J. Mech. Des. 2021, 143, 043501. [CrossRef]

8. Su, Q.; Lai, S.J. 3D geometric constraint analysis and its application on the spatial assembly sequence planning. Int. J. Prod. Res. 2010, 48, 1395-1414. [CrossRef]

9. Goka, E.; Homri, L.; Beaurepaire, P.; Dantan, J.Y. Statistical Tolerance Analysis of Over-Constrained Mechanical Assemblies with Form Defects Considering Contact Types. J. Comput. Inf. Sci. Eng. 2019, 19, 021010. [CrossRef]

10. Zhao, D.; Wang, G.; Hui, J.; Hou, W.; Evans, R.D. A precision analysis method for the kinematic assembly of complex products based on equivalence of deviation source. Assem. Autom. 2020, 40, 447-460. [CrossRef]

11. Li, J.; Ran, Y.; Wang, H.W.; Mu, Z.Y.; Zhang, G.B. A tolerance analysis method for complex mechanical products based on meta-action unit. Int. J. Adv. Manuf. Technol. 2021, 117, 1377-1393. [CrossRef]

12. Wang, K.; Liu, D.; Liu, Z.Y.; Wang, Q.; Tan, J.R. An assembly precision analysis method based on a general part digital twin model. Robot Comput. Integr. Manuf. 2021, 68, 102089. [CrossRef]

13. McKenna, V.; Jin, Y.; Murphy, A.; Morgan, M.; Fu, R.; Qin, X.D.; McClory, C.; Collins, R.; Higgins, C. Cost-oriented process optimisation through variation propagation management for aircraft wing spar assembly. Robot Comput. Integr. Manuf. 2019, 57, 435-451. [CrossRef]

14. Mei, B.; Zhu, W.D. Accurate positioning of a drilling and riveting cell for aircraft assembly. Robot Comput. Integr. Manuf. 2021, 69, 102112. [CrossRef]

15. Guo, J.; Liu, Z.; Li, B.; Hong, J. Optimal tolerance allocation for precision machine tools in consideration of measurement and adjustment processes in assembly. Int. J. Adv. Manuf. Technol. 2015, 80, 1625-1640. [CrossRef]

16. Sun, W.; Mu, X.K.; Sun, Q.C.; Sun, Z.Y.; Wang, X.B. Analysis and optimization of assembly precision-cost model based on 3D tolerance expression. Assem. Autom. 2018, 38, 497-510. [CrossRef]

17. Cao, Y.J.; Li, X.; Zhang, Z.X.; Shang, J.Z. Dynamic prediction and compensation of aerocraft assembly variation based on state space model. Assem. Autom. 2015, 35, 183-189. [CrossRef]

18. Li, H.; Zhu, H.P.; Li, P.G.; He, F. Tolerance analysis of mechanical assemblies based on small displacement torsor and deviation propagation theories. Int. J. Adv. Manuf. Technol. 2014, 72, 89-99. [CrossRef]

19. Speckhart, F.H. Calculation of tolerance based on a minimum cost approach. J. Eng. Ind. 1972, 94, 447-453. [CrossRef]

20. Singh, P.K.; Jain, P.K.; Jain, S.C. Simultaneous optimal selection of design and manufacturing tolerances with different stack-up conditions using genetic algorithms. Int. J. Prod. Res. 2003, 41, 2411-2429. [CrossRef]

21. Wang, G.D.; Yang, Y.; Wang, W.; Si-Chao, L.V. Variable coefficients reciprocal squared model based on multi-constraints of aircraft assembly tolerance allocation. Int. J. Adv. Manuf. Technol. 2016, 82, 227-234. [CrossRef] 
22. Sanz-Lobera, A.; Gomez, E.; Perez, J.; Sevilla, L. A proposal of cost-tolerance models directly collected from the manufacturing process. Int. J. Prod. Res. 2016, 54, 4584-4598. [CrossRef]

23. Dong, Z.; Hu, W. Optimal process sequence identification and optimal process tolerance assignment in computer-aided process planning. Comput. Ind. 1991, 17, 19-32. [CrossRef]

24. Jeang, A.; Leu, E. Robust tolerance design by computer experiment. Int. J. Prod. Res. 1999, 37, 1949-1961. [CrossRef]

25. Lu, C.; Fuh, J.Y.H.; Wong, Y.S. Evaluation of product assemblability in different assembly sequences using the tolerancing approach. Int. J. Prod. Res. 2006, 44, 5037-5063. [CrossRef]

26. Hallmann, M.; Schleich, B.; Wartzack, S. Process and machine selection in sampling-based tolerance-cost optimisation for dimensional tolerancing. Int. J. Prod. Res. 2021, 59, 1-16. [CrossRef]

27. Wu, H.R.; Li, X.X.; Sun, F.C.; Zheng, H.L.; Zhao, Y.X. Optimization design method of machine tool static geometric accuracy using tolerance modeling. Int. J. Adv. Manuf. Technol. 2021, 116, 1-17. [CrossRef]

28. Lee, K.K.; Ro, Y.C.; Han, S.H. Tolerance Optimization of a Lower Arm by using Genetic Algorithm and Process Capability Index. Int. J. Precis. Eng. Manuf. 2014, 15, 1001-1007. [CrossRef]

29. Prabhaharan, G.; Asokan, P.; Rajendran, S. Sensitivity-based conceptual design and tolerance allocation using the continuous ants colony algorithm (CACO). Int. J. Adv. Manuf. Technol. 2005, 25, 516-526. [CrossRef]

30. Zhang, Y.M.; Ji, S.T.; Zhao, J.; Xiang, L.J. Tolerance analysis and allocation of special machine tool for manufacturing globoidal cams. Int. J. Adv. Manuf. Technol. 2016, 87, 1597-1607. [CrossRef]

31. Wang, Y.; Li, L.; Hartman, N.W.; Sutherland, J.W. Allocation of assembly tolerances to minimize costs. CIRP Ann. Manuf. Technol. 2019, 68, 13-16. [CrossRef]

32. Kumar, D.V.; Ravindran, D.; Lenin, N.; Kumar, M.S. Tolerance allocation of complex assembly with nominal dimension selection using Artificial Bee Colony algorithm. Proc. Inst. Mech. Eng. Part C J. Mech. Eng. Sci. 2019, 233, 18-38. [CrossRef]

33. Kumar, M.S.; Kannan, S.M.; Jayabalan, V. A new algorithm for optimum tolerance allocation of complex assemblies with alternative processes selection. Int. J. Adv. Manuf. Technol. 2009, 40, 819-836. [CrossRef]

34. Forouraghi, B. Optimal tolerance allocation using a multiobjective particle swarm optimizer. Int. J. Adv. Manuf. Technol. 2009, 44, 710-724. [CrossRef]

35. Zhang, C.; Wang, H.P. Integrated tolerance optimisation with simulated annealing. Int. J. Adv. Manuf. Technol. 1993, 8, 167-174. [CrossRef]

36. Coelho, L.D. Self-organizing migration algorithm applied to machining allocation of clutch assembly. Math. Comput. Simul. 2009, 80, 427-435. [CrossRef]

37. Kumar, L.R.; Padmanaban, K.P.; Kumar, S.G.; Balamurugan, C. Design and optimization of concurrent tolerance in mechanical assemblies using bat algorithm. J. Mech. Sci. Technol. 2016, 30, 2601-2614. [CrossRef]

38. Wu, Z.J.; Liu, T.; Gao, Z.B.; Cao, Y.L.; Yang, J.X. Tolerance design with multiple resource suppliers on cloud-manufacturing platform. Int. J. Adv. Manuf. Technol. 2016, 84, 335-346. [CrossRef]

39. Balamurugan, C.; Saravanan, A.; Babu, P.D.; Jagan, S.; Narasimman, S.R. Concurrent optimal allocation of geometric and process tolerances based on the present worth of quality loss using evolutionary optimisation techniques. Res. Eng. Des. 2017, 28, 185-202. [CrossRef]

40. Kumar, L.R.; Padmanaban, K.P.; Balamurugan, C. Optimal tolerance allocation in a complex assembly using evolutionary algorithms. Int. J. Simul. Model 2016, 15, 121-132. [CrossRef]

41. Sanfilippo, E.M.; Belkadi, F.; Bernard, A. Ontology-based knowledge representation for additive manufacturing. Comput. Ind. 2019, 109, 182-194. [CrossRef]

42. Xing, X.J.; Zhong, B.T.; Luo, H.B.; Li, H.; Wu, H.T. Ontology for safety risk identification in metro construction. Comput. Ind. 2019, 109, 14-30. [CrossRef]

43. He, W.; Wang, F.K.; Akula, V. Managing extracted knowledge from big social media data for business decision making. J. Knowl. Manag. 2017, 21, 275-294. [CrossRef]

44. Gao, J.; Bernard, A. An overview of knowledge sharing in new product development. Int. J. Adv. Manuf. Technol. 2018, 94, 1545-1550. [CrossRef]

45. France-Mensah, J.; O’Brien, W.J. A shared ontology for integrated highway planning. Adv. Eng. Inform. 2019, 41, 100929. [CrossRef]

46. Peng, G.Z.; Wang, H.W.; Zhang, H.M.; Zhao, Y.W.; Johnson, A.L. A collaborative system for capturing and reusing in-context design knowledge with an integrated representation model. Adv. Eng. Inform. 2017, 33, 314-329. [CrossRef]

47. Sanfilippo, E.M.; Borgo, S. What are features? An ontology-based review of the literature. Comput. Aided Des. 2016, 80, 9-18. [CrossRef]

48. Sudarsan, R.; Fenves, S.J.; Sriram, R.D.; Wang, F. A product information modeling framework for product lifecycle management. Comput. Aided Des. 2005, 37, 1399-1411. [CrossRef]

49. Barbau, R.; Krima, S.; Rachuri, S.; Narayanan, A.; Fiorentini, X.; Foufou, S.; Sriram, R.D. OntoSTEP: Enriching product model data using ontologies. Comput. Aided Des. 2012, 44, 575-590. [CrossRef]

50. Sarigecili, M.I.; Roy, U.; Rachuri, S. Interpreting the semantics of GD\&T specifications of a product for tolerance analysis. Comput. Aided Des. 2014, 47, 72-84. [CrossRef]

51. Zhong, Y.R.; Qin, Y.C.; Huang, M.F.; Lu, W.L.; Gao, W.X.; Du, Y.L. Automatically generating assembly tolerance types with an ontology-based approach. Comput. Aided Des. 2013, 45, 1253-1275. [CrossRef] 
52. Qin, Y.C.; Lu, W.L.; Qi, Q.F.; Li, T.K.; Huang, M.F.; Scott, P.J.; Jiang, X.Q. Explicitly representing the semantics of composite positional tolerance for patterns of holes. Int. J. Adv. Manuf. Technol. 2017, 90, 2121-2137. [CrossRef]

53. Lu, W.L.; Qin, Y.C.; Liu, X.J.; Huang, M.F.; Zhou, L.P.; Jiang, X.Q. Enriching the semantics of variational geometric constraint data with ontology. Comput. Aided Des. 2015, 63, 72-85. [CrossRef] 